\section{(6) OPEN ACCESS}

\title{
Toll-like receptor 7 governs interferon and inflammatory responses to rhinovirus and is suppressed by IL-5-induced lung eosinophilia
}

\author{
Luke Hatchwell, 1,2 Adam Collison, 1,2 Jason Girkin, 1,2 Kristy Parsons, 2,3 \\ Junyao Li, ${ }^{1,2,4}$ Jie Zhang, ${ }^{4}$ Simon Phipps, ${ }^{5}$ Darryl Knight, ${ }^{2}$ Nathan W Bartlett, ${ }^{6}$ \\ Sebastian L Johnston, ${ }^{6}$ Paul S Foster, ${ }^{2}$ Peter A B Wark, ${ }^{2,3}$ Joerg Mattes ${ }^{1,2,7}$
}

\begin{abstract}
- Additional material is published online only. To view please visit the journal online (http://dx.doi.org/10.1136/ thoraxjn-2014-205465).
\end{abstract}

For numbered affiliations see end of article.

\section{Correspondence to Professor Joerg Mattes, Department of Paediatric Respiratory and Sleep Medicine, Newcastle Children's Hospital, Locked Bag 1, Newcastle, NSW 2305, Australia; joerg.mattes@newcastle.edu. au}

Received 25 March 2014 Revised 27 April 2015 Accepted 15 May 2015 Published Online First 24 June 2015

\section{ABSTRACT}

Background Asthma exacerbations represent a significant disease burden and are commonly caused by rhinovirus (RV), which is sensed by Toll-like receptors (TLR) such as TLR7. Some asthmatics have impaired interferon (IFN) responses to RV, but the underlying mechanisms of this clinically relevant observation are poorly understood.

Objectives To investigate the importance of intact TLR7 signalling in vivo during RV exacerbation using mouse models of house dust mite (HDM)-induced allergic airways disease exacerbated by a superimposed RV infection.

Methods Wild-type and TLR7-deficient $\left(T / r 7^{-/-}\right)$BALB/ $C$ mice were intranasally sensitised and challenged with HDM prior to infection with RV1B. In some experiments, mice were administered recombinant IFN or adoptively transferred with plasmacytoid dendritic cells ( $p D C)$. Results Allergic T/r $7^{-/-}$mice displayed impaired IFN release upon RV1B infection, increased virus replication and exaggerated eosinophilic inflammation and airways hyper reactivity. Treatment with exogenous IFN or adoptive transfer of TLR7-competent pDCs blocked these exaggerated inflammatory responses and boosted IFN $\gamma$ release in the absence of host TLR7 signalling. TLR7 expression in the lungs was suppressed by allergic inflammation and by interleukin (IL)-5-induced eosinophilia in the absence of allergy. Subjects with moderate-to-severe asthma and eosinophilic but not neutrophilic airways inflammation, despite inhaled steroids, showed reduced TLR7 and IFN 2 2/3 expression in endobronchial biopsies. Furthermore, TLR7 expression inversely correlated with percentage of sputum eosinophils.

Conclusions This implicates IL-5-induced airways eosinophilia as a negative regulator of TLR7 expression and antiviral responses, which provides a molecular mechanism underpinning the effect of eosinophiltargeting treatments for the prevention of asthma exacerbations.
CrossMark

To cite: Hatchwell $L_{\text {, }}$ Collison A, Girkin J, et al. Thorax 2015;70:854-861.

\section{INTRODUCTION}

Asthma is a complex and heterogenic inflammatory disease of the airways with increasing global prevalence. The most common trigger for asthma symptoms is immune cell activation against innocuous antigens (allergens) and respiratory viral infections. ${ }^{1}$

\section{Key messages}

What is the key question?

- What underlies impaired interferon responses to rhinovirus in asthmatics.

What is the bottom line?

- Suppression of Toll-like receptor (TLR)7 by interleukin-5-induced lung eosinophilia impairs interferon responses to rhinovirus, leading to exaggerated inflammatory responses and exacerbation.

\section{Why read on?}

- This provides a mechanism whereby therapies modulating TLR7 or targeting eosinophils may ameliorate virus-induced asthma exacerbations.

Upon antigen exposure, cytokines such as thymic stromal lymphopoietin (TSLP), granulocytemacrophage colony-stimulating factor, interleukin (IL)-25, IL-33 and tumour necrosis factor-related apoptosis-inducing ligand are released by the airway epithelium, resulting in the activation of innate immune cells that promote T helper $2\left(\mathrm{~T}_{\mathrm{H}} 2\right)$ cell differentiation, leading to the release of $\mathrm{T}_{\mathrm{H}} 2$ cytokines such as IL-4, IL-5 and IL-13. ${ }^{1}{ }^{2}$ IL-13 is a potent inducer of airway hyper reactivity (AHR) and mucus production in a signal transducer and activator of transcription-6 (STAT6)-dependent manner. ${ }^{3}$ IL-5 regulates maturation of eosinophils in the bone marrow and in concert with chemokines such as eotaxins the recruitment of these cells into the airways. ${ }^{4}$ Thus, $\mathrm{T}_{\mathrm{H}} 2$ cell activation underpins many clinical phenotypes including allergic and eosinophilic asthma. ${ }^{5}$ The proportion of asthmatics with high eosinophil numbers in their airways represent the majority of patients, and some of those unresponsive to corticosteroids have severe therapy-refractory asthma with a disproportionally large burden of disease. Importantly, they are also prone to asthma exacerbations, which can be partially alleviated by therapeutics that block IL-4, IL-5 or IL-13 and reduce eosinophilic inflammation in the lungs. ${ }^{5}$ However, the molecular mechanisms that link $\mathrm{T}_{\mathrm{H}}$ 2-induced eosinophilia with susceptibility to exacerbation are yet to be defined. 
Viral respiratory infections are detected in up to $85 \%$ of asthma exacerbations and two-thirds of those are caused by rhinoviruses (RV). ${ }^{6}$ Notably a group of asthmatics have impaired release of innate interferons (IFN $\alpha$, IFN $\beta$ and IFN $\lambda$ ) upon experimental RV infection and IFN $\lambda$ inversely correlated with induced sputum eosinophils on day 3 of acute infection. ${ }^{7} 8$ IFN $\lambda 2 / 3$ inversely correlated with induced sputum eosinophils on day 3 of the acute infection $(\mathrm{r}=-0.53, \mathrm{p}=0.05)$, when subjects were experimentally infected with RV16 in vivo. ${ }^{8} \mathrm{RV}$ is sensed by a limited number of pattern recognition receptors, including Toll-like receptors (TLRs) and retinoid acid-induced gene 1 like receptors. TLR3 and TLR7 are endosomally localised and recognise viral nucleic acids, critically regulating antiviral IFN production. Recent studies have shown that impaired TLR3 function does not affect RV replication in vivo and TLR3 expression is not reduced in asthmatics. ${ }^{9}{ }^{10}$ The in vivo role of functional TLR7 signalling in mounting antiviral responses to RV has yet to be determined. TLR7 is widely expressed in innate immune cells such as dendritic cells (DCs) and macrophages, as well as in structural lung cells, including airway epithelia. ${ }^{11}$ Treatment of mice with TLR7 agonists leads to a long-lasting protection from the development of allergic airways disease (AAD) and TLR7 activation also reduced airway smooth muscle contractility in mice. ${ }^{12}$ We and others have found reduced IFN $\alpha$ and IFN $\lambda$ release upon TLR7 stimulation of peripheral blood mononuclear cells from asthmatics and a trend towards lower TLR7 expression was observed in bronchoalveolar lavage fluid (BALF) cells from asthmatics. ${ }^{9}$ However, the role of TLR7 in RV-induced exacerbation of AAD has yet to be fully elucidated.

\section{METHODS}

\section{Patient biopsies}

Endobronchial biopsies were obtained by bronchoscopy with samples taken from third-generation bronchi. Biopsies were stored in RNALater (Ambion) at $-20^{\circ} \mathrm{C}$ until needed. RNA was extracted following the miRNeasy Mini Handbook (Qiagen)purification of total RNA from tissue via homogenisation (Qiashredder). RNA was quantified by spectrophotometry (NanoDrop) and $200 \mathrm{ng}$ of extracted RNA reverse transcribed using High Capacity cDNA Reverse Transcription Kit (Applied Biosystems; ABI). The inflammatory phenotype was determined by induced sputum count, and patients with a RAST ImmunoCAP Specific IgE-positive blood test were designated as atopic.

\section{Animals}

Wild-type (WT), Tlr $7^{-/-}, T l r 4^{-/-}, M y D 88^{-/-}$, Stat $^{-/-}$and $I L-5$ transgenic mice, all on a BALB/c background (6-14 weeks of age), were obtained from Australian BioResources (Moss Vale, Australia) and housed in approved containment facilities within the HMRI Building, University of Newcastle (Newcastle, Australia). Mice had ad libitum access to food and water under a $12 \mathrm{~h}$ light and dark cycle. All experiments were approved by the Animal Care and Ethics Committee of the University of Newcastle.

\section{Induction of AAD and RV-induced exacerbation}

$\mathrm{AAD}$ and RV-induced exacerbation were performed as previously described. ${ }^{13}$ Briefly, lyophilised crude house dust mite (HDM) (Dermatophagoides pteronyssinus) extract (Greer Laboratories) was resuspended in sterile saline (SAL) and intranasally delivered to mice under light isoflurane anaesthesia. Sensitisation on days 0,1 and 2 (HDM $50 \mu \mathrm{g} / 50 \mu \mathrm{L}$ ) was followed by daily challenge (HDM $5 \mu \mathrm{g} / 50 \mu \mathrm{L}$ ) on days 14,15 , 16 and 17 to induce AAD. On day 18, mice were euthanased via pentobarbital sodium overdose (Virbac) and samples collected. In other experiments, mice were intranasally infected with live minor group RV (RV1B), $50 \mu \mathrm{L}$ containing $5 \times 10^{6}$ virions median tissue culture infective dose $\left(\operatorname{TCID}_{50}\right)$ or UV-inactivated RV1B $24 \mathrm{~h}$ after last HDM exposure to exacerbate pre-existing AAD. Samples were collected $24 \mathrm{~h}$ after RV1B infection.

\section{Administration of recombinant cytokines and LPS}

Naive mice were intranasally administered recombinant mouse IL-13 (15 $\mu \mathrm{g} / 35 \mu \mathrm{L}$; Biolegend), IL-5 (15 $\mu \mathrm{g} / 35 \mu \mathrm{L}$; Biolegend) or a low or high dose $(0.12 \mu \mathrm{g} / 50 \mu \mathrm{L}$ and $10 \mu \mathrm{g} / 50 \mu \mathrm{L}$, respectively) of lipopolysaccharide (LPS) (Escherichia coli, 0111:B4; Sigma-Aldrich), with samples collected 24 h later. HDM-sensitised and challenged $T l r 7^{-/-}$mice, which also received live RV1B, were intranasally administered either recombinant mouse IFNo2 (10 $000 \mathrm{IU} / 50 \mu \mathrm{L} ; \quad$ eBioscience), IFN $\beta 1 \quad(10000 \mathrm{IU} / 50 \mu \mathrm{L}$; Biolegend), IFN $\lambda 2\left(1 \mu \mathrm{g} / 50 \mu \mathrm{L}\right.$; R\&D Systems) ${ }^{14}$ or a vehicle control (phosphate buffered saline) on day 18 ( $2 \mathrm{~h}$ following RV1B infection). Mice were sacrificed $24 \mathrm{~h}$ postinfection and samples collected.

\section{AHR measurement}

AHR was measured as previously described. ${ }^{15}$ Briefly, AHR was invasively assessed in separate groups of anaesthetised mice by measurement of total lung resistance and dynamic compliance (Buxco). Mice were mechanically ventilated, and AHR to nebulised methacholine (increased lung resistance) was expressed as a percentage change from control (baseline).

\section{Analysis of lung inflammation}

BALF was collected and analysed as previously described. ${ }^{16}$ Enumeration of peribronchial/perivascular eosinophils and PAS-positive cells was performed as previously described. ${ }^{17}$

\section{Flow cytometry}

Single lung cell suspensions were prepared and stained as previously described. ${ }^{18}$ Antibodies used were FITC-anti-TCR $\beta$ chain (BD, cat. no. 553171, clone H57-597), PE-anti-CD4 (BD, cat. no. 553652, clone H129.19), PerCP-anti-CD8a (BD, cat. no. 561092, clone 53-6.7), PerCP-Cy5.5-anti-CD11b (BD, cat. no. 561092, clone M1/70), FITC-anti-CD11c (BD, cat. no. 553801, clone HL3), PE-anti-MHCII (eBioscience, cat. no. 12-5321, clone M5/114.15.2), all at 1:15 dilution. Positive cells were identified using a FACSCanto (BD) by the following criteria: mDCs-CD11 ${ }^{+} \mathrm{CD}_{11 \mathrm{c}^{+}} \mathrm{MHCII}^{+} ; \mathrm{T}$ cells-TCR $\beta$ chain ${ }^{+}$ with $\mathrm{CD}^{+}{ }^{+}$or $\mathrm{CD} 8 \mathrm{a}^{+}$. Data analysed with BD FACsdiva.

\section{Quantitative RT-PCR}

Trachea and lungs were extracted from euthanased mice and forceps used to separate the airways from the parenchyma by blunt dissection. ${ }^{19}$ Total mRNA was extracted using TRIzol (Ambion; Carlsbad, USA). cDNA was generated via reverse transcription using BioScript (Bioline; Alexandria, Australia). Quantitative PCRs (qPCR) were performed on cDNA generated from mouse airway tissue and human endobronchial biopsies with SYBR Green (Invitrogen; Mulgrave, Australia) using primers detailed in online supplementary table S1. CTs of the genes of interest were referenced HPRT or GAPDH for mouse and human tissue, respectively. All steps were performed according to manufacturer's instructions. 


\section{Generation of BM-derived $\mathrm{pDCs}$ and adoptive transfer}

WT and TLR7-deficient bone marrow-derived plasmacytoid DCs (pDCs) were generated as previously described. ${ }^{20}$ pDCs were resuspended at $1 \times 10^{7}$ cells $/ \mathrm{mL}$ and $0.5 \times 10^{6} \mathrm{pDC}$ were administered intranasally to allergic $T l r 7^{-/-}$mice, $2 \mathrm{~h}$ prior to RV1B infection.

\section{Quantification of lung cytokines and IFNs}

A single lung lobe from each mouse was excised and snap frozen before being homogenised in buffers recommended in the manufacturer's instructions. Levels of IL-5, IL-13, IFN $\gamma$ (BD Biosciences; PharMingen), IFN $\alpha$, IFN $\beta$ (Verikine; PBL Assay Science), CCL7/MCP3 and IFN $22 / 3$ (R\&D Systems) were determined in clarified lung lysates by ELISA. CCL2/MCP1, CCL3/ MIP1 $\alpha$, CCL4/MIP1 $\beta$, CXCL9/MIG and CXCL10/IP10 were measured by employing a Multiplex Immunoassay (Millipore). All concentrations were normalised to lung weight.

\section{pDC culture and $\mathrm{TCID}_{50}$}

Primary mouse pDCs were isolated from pooled WT or TLR7-deficient spleens via mechanical dissociation and isolation with PDCA-1 magnetic beads on an AutoMACS platform (Miltenyi Biotec) according to manufacturer's instructions. Following two isolation runs, positively selected cells were seeded into 96-well plates at $1 \times 10^{6}$ cells/well and cultured in the absence or presence of RV1B (multiplicity of infection $=5$ ). ${ }^{20}$ Supernatant was harvested $48 \mathrm{~h}$ postinfection and IFN production assessed with ELISA. Number of live RV1B virions was determined by standard TCID $_{50}$ serial dilution on Hela-H1 cells, calculated via the Spearman-Karber method.

\section{Statistical analysis}

The significance of differences between groups was analysed using Student's t test, Mann-Whitney test, analysis of variance or Kruskal-Wallis with Dunn's test for multiple comparisons as appropriate using Graphpad Prism 6. A value of $p<0.05$ is reported as significant. Data are expressed as mean \pm SEM.

\section{RESULTS}

To investigate TLR7 as a regulator of AAD and antiviral responses in vivo, we repeatedly challenged WT and TLR7-deficient $\left(T l r 7^{-/}\right)$mice with HDM, then superimposed a RV infection to exacerbate their established AAD as described previously. ${ }^{15}$ Exposure of allergic Tlr $7^{-1-}$ mice to RV, compared with WT controls, resulted in impaired production of type I $(\alpha, \beta)$, II $(\gamma)$ and III $(\lambda)$ IFNs with higher RV replication in the lower airways (figure 1A,B). This was despite a lack of an increase in type II $(\gamma)$ and III $(\lambda)$ IFNs to RV infection in allergic WT mice. Deficient IFN responses coincided with exaggerated AHR (figure 1C), eosinophilic airways inflammation (figure 1D,E), accumulation of $\mathrm{CD} 4+, \mathrm{CD} 8+$ and myeloid (m) DCs but not pDCs in the lungs (figure 1F), and production IL-5 and CCL11 (eotaxin-1) (figure 1G). We also observed increased levels of the $\mathrm{T}_{\mathrm{H}}$ 2-priming cytokines IL-25 and TSLP in allergic $T l r 7^{-/-}$mice infected with RV (see online supplementary figure S1). CCL2, CCL3, CCL4 and CCL7 but not CXCL9 and CXCL10 were also increased in the absence of TLR7 signalling (see online supplementary figure S2). There was no change in IL-13 levels or numbers of mucus-producing cells (see online supplementary figure S3a), suggesting a novel and critical role of TLR7 signalling in RV-induced asthma exacerbation.
Allergic $T l r 7^{-/-}$mice received recombinant type I and III IFNs $2 \mathrm{~h}$ after infection with RV. Notably, one dose of IFN $\alpha 2, \beta$ or $\lambda 2$ suppressed RV-induced eosinophilic inflammation (figure 2A), as well as IL-5 and CCL11 production (figure 2B) but not IL-13 (see online supplementary figure S3b). All IFN treatments induced IFN- $\gamma$ in the lung (figure 2C) and limited RV replication (figure 2D). There was no effect of IFN treatment on TSLP, IL-25 or IL-33 expression (data not shown). Thus IFN treatment promotes IFN- $\gamma$ release and impairs IL-5 and CCL11 production and RV replication in the absence of TLR7.

pDCs release large quantities of IFN during viral infection, and here we show that splenic pDCs exposed to RV in vitro display impaired release of IFN- $\alpha$ and IFN- $\beta$ but not $\lambda 2 / 3$ in the absence of TLR7 (figure 3A). Adoptive transfer of TLR7-expressing pDCs into allergic $T l r 7^{-/-}$mice re-established IFN $\alpha$, IFN $\beta$ and IFN $\gamma$ but not IFN $\lambda$ release in the lungs upon RV infection and limited RV replication as compared to allergic $T l r 7^{-/-}$mice that received TLR7-deficient pDCs (figure 3B, C). Transfer of WT pDCs also limited RV-induced AHR (figure 3D), eosinophilic airways inflammation (figure $3 \mathrm{E}$ ) and production of IL-5 and CCL11 (figure 3F) but not IL-13 release (see online supplementary figure S3c). Thus adoptive transfer of TLR7-expressing pDCs-like exogenous IFN treatment-promotes IFN $\gamma$ release and impairs eosinophilic airways inflammation and RV replication in the absence of TLR7.

TLR7 expression was assessed in a number of knockout mice strains as it was suppressed in allergic WT mice with $\mathrm{T}_{\mathrm{H}}$ 2-mediated AAD (figure 4A). Notably this allergen-induced reduction in TLR7 expression was not observed in TLR4 $\left(\mathrm{Tlr}^{-/-}\right)$, MyD88 $\left(\mathrm{Myd} 88^{-/-}\right)$or STAT6 $\left(\right.$Stat $\left.^{-/-}\right)$-deficient mice, suggesting that intact $\mathrm{T}_{\mathrm{H}} 2$-promoting signalling pathways and the presence of eosinophilic airways inflammation are required for suppression of TLR7. To investigate the specific role of $\mathrm{T}_{\mathrm{H}} 2$ cytokines in this in vivo observation, we delivered one dose of recombinant IL-13 or IL-5 intranasally to WT mice and compared those responses to ones challenged with LPS (a TLR4 agonist). Interestingly, in mice that constitutively overexpress IL-5 (IL-5 ${ }^{T g / T g}$ mice) TLR7 expression was significantly reduced (figure 4B) and was associated with accumulation of eosinophils in the airways in the absence of allergy (figure 4D). Intranasal administration of one dose of IL-5 or IL-13, however, had no effect on TLR7 expression or eosinophil recruitment although IL-13 did induce AHR and increased expression of Muc5AC (data not shown). In addition, one low or high dose of LPS increased TLR7 expression and resulted in the accumulation of neutrophils but not eosinophils in the lungs (figure 4C,D). Thus eosinophilic airways inflammation due to chronic IL-5 release is associated with a reduction in TLR7 expression while a single administration of IL- 5 or IL-13 had no effects on TLR7.

We next analysed TLR7 expression in bronchial biopsies collected from healthy subjects and patients with moderate-to-severe persistent asthma (clinical and demographic data in online supplementary table S2). Importantly, asthmatics with an eosinophilic airways inflammation, as determined by bronchial lavage, ${ }^{21}$ displayed significantly reduced TLR7 (figure 4E) and IFN $\lambda 2 / 3$ expression (figure $4 \mathrm{~F}$ ), independent of atopic status (figure 4G). Furthermore, levels of TLR7 expression positively correlated with IFN $\alpha$ and $\lambda 2 / 3$ expression (figure 4I-K) and inversely correlated with percentage of sputum eosinophils (figure $4 \mathrm{H})$ but not macrophages or neutrophils $\left(r_{s}=0.04\right.$; $\mathrm{p}=0.81 ; \mathrm{n}=33$ and $\mathrm{r}_{\mathrm{s}}=0.31 ; \mathrm{p}=0.08 ; \mathrm{n}=33$, respectively). These results suggest that suppression of TLR7 and IFN 

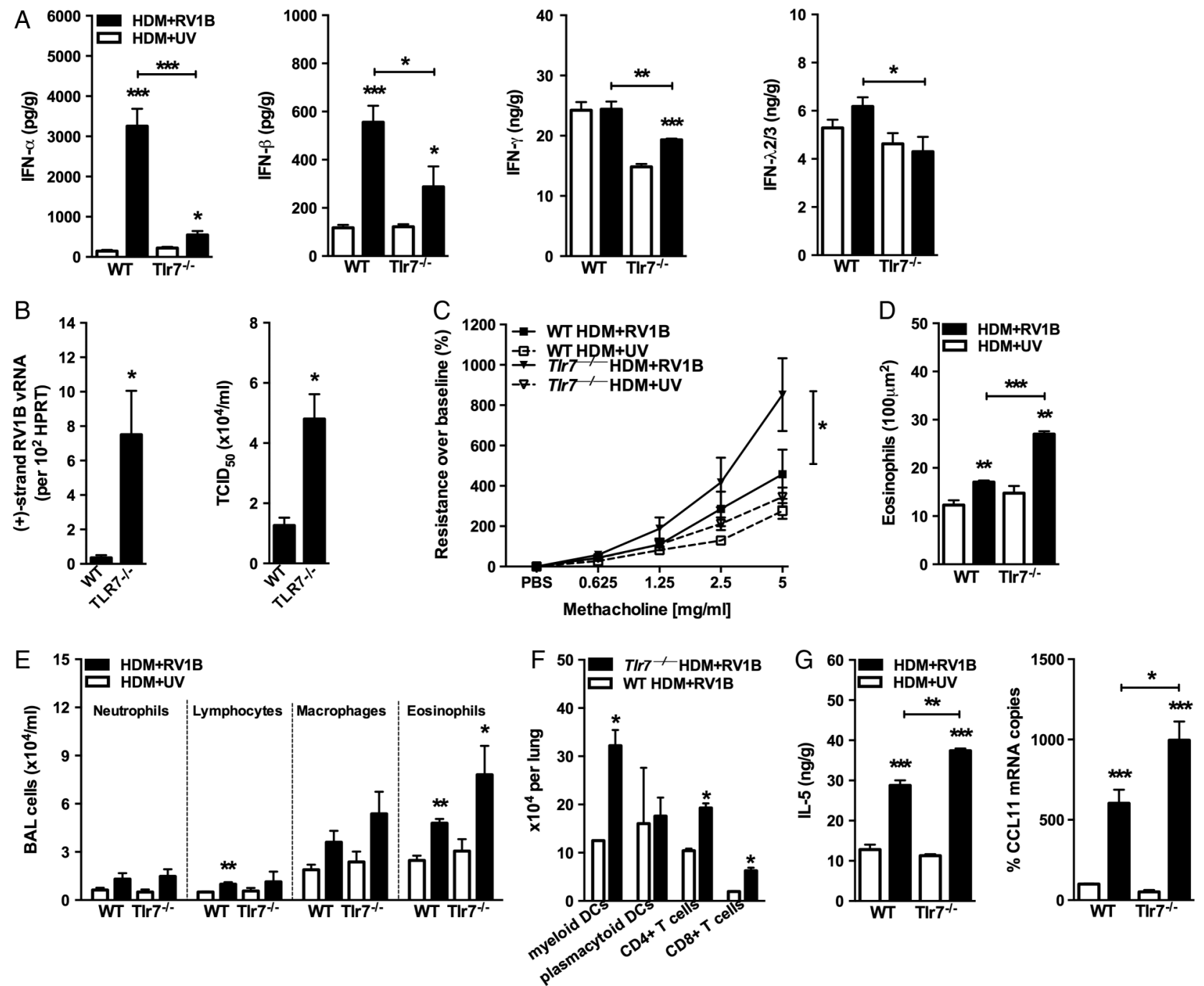

Figure 1 Toll-like receptor (TLR)7-deficient mice experience exaggerated rhinovirus (RV)-induced exacerbation. Allergic wildtype (WT) and TLR7-deficient mice were inoculated with live or UV-inactivated RV1B, samples collected $24 \mathrm{~h}$ post infection. Levels of interferons (A) and interleukin (IL)-5 (G) in clarified lung homogenates as assessed by ELISA. (B) Lung viral titre in infected mice was quantified via quantitative RT-PCR (qRT-PCR) and $50 \%$ tissue culture infectious dose $\left(\mathrm{TCID}_{50}\right)$. (C) Total lung resistance presented as percentage change in response to methacholine $(\mathrm{n}=4-6$ mice per group). Peribronchial/perivascular eosinophils and cellular infiltrates in bronchoalveolar lavage (BAL) fluid enumerated by light microscopy (D and E). (F) Numbers of lung mDCs and CD4+/CD8+ T cells quantified by flow cytometry. (G) CCL11 expression in lower airway tissue enumerated by qRT-PCR and expressed as \% increase over WT house dust mite (HDM)+UV group. Results are mean $\pm S E M(n=3-6$ mice per group) and are representative of two independent experiments. ${ }^{*} p<0.05,{ }^{* *} p<0.01,{ }^{* * *} p<0.001$ as compared to strain-matched HDM+UV group or otherwise indicated determined by students $t$ test except for (C) where analysis of variance was used to compare RI curves.

expression in moderate-to-severe asthmatics is specifically tied to eosinophilic airways inflammation in this cohort.

\section{DISCUSSION}

Impaired innate IFN responses to respiratory viruses have been proposed as one mechanism underlying the clinical observation of asthmatics being susceptible to RV-induced exacerbation. ${ }^{78}$ Innate IFN responses are instigated by a limited number of pattern recognition receptors, such as TLR7 whose cognate ligands include the RV viral genome. ${ }^{10}{ }^{11}$ Experiments in human immortalised epithelial cell lines (BEAS-2B) have supported a role of TLR3 in mediating an antiviral and anti-inflammatory response. ${ }^{9}$ However, TLR3 expression in the airways ${ }^{22}$ and TLR3-induced responses did not vary in PBMCs derived from asthmatic or atopic patients by comparison to healthy subjects. ${ }^{22}$ We show here, for the first time in vivo, that a lack of TLR7 signalling under conditions that model a viral asthma exacerbation leads to impaired IFN production and exaggerated $\mathrm{T}_{\mathrm{H}}$ 2-driven inflammation. Our findings, such as increased levels of the $T_{H}$ 2-priming cytokines IL-25, IL-33 and TSLP in $T l r 7^{-/-}$mice, mirror those published by Kaiko et al, ${ }^{23}$ infecting non-allergic mice with mouse pneumovirus, a mouse pathogen similar to human respiratory syncytial virus. These studies suggest that intact TLR7 signalling is required for sufficient IFN induction and restrainment of proinflammatory responses in the lung.

As TLR7 activation is upstream of IFN production, we hypothesised that administration of exogenous IFN would protect allergic $T l r 7^{-1-}$ mice from RV-induced exacerbation. We observed that treatment with type I or III IFNs resulted in the $T l r 7^{-/-}$mice developing a suppressed phenotype similar to that of the WT controls, whose TLR7 signalling is active. All IFN treatments induced the release of IFN $\gamma$ in the lung, with type I and III IFNs promoting themselves exclusively, indicative of their known distinct signalling pathways. ${ }^{14}$ 

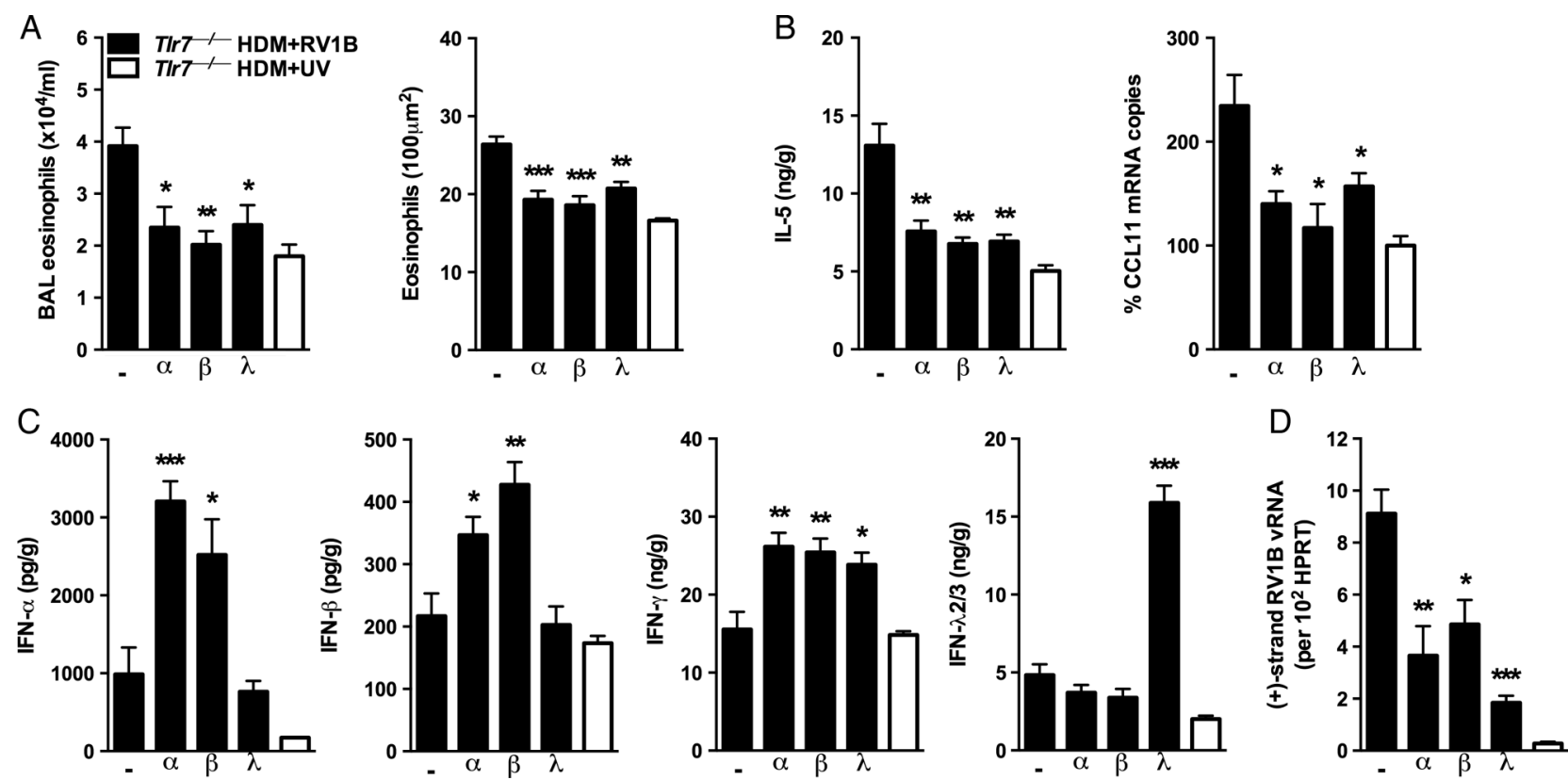

Figure 2 Exogenous interferon can protect Toll-like receptor (TLR)7-deficient mice from rhinovirus (RV)-induced exacerbation. Exacerbated TLR7-deficient mice received recombinant interferon (IFN) $\alpha 2(\alpha)$, IFN $\beta(\beta)$, IFN $\lambda 2(\lambda)$ or a vehicle control $(-) 2 \mathrm{~h}$ post-RV1B infection on day 18. Samples were collected $24 \mathrm{~h}$ post infection. (A) Eosinophils present in bronchoalveolar lavage (BAL) fluid and per $100 \mu \mathrm{m}^{2}$ of lung tissue. Levels of interleukin (IL)-5 (B) and interferons (C) in clarified lung homogenates as assessed by ELISA. CCL11 expression (B) and viral RV1B RNA (D) in lower airway tissue was quantified by quantitative RT-PCR. Results are mean \pm SEM ( $n=3-7$ mice per group) and are representative of two independent experiments. ${ }^{*} p<0.05,{ }^{* *} p<0.01,{ }^{* * *} p<0.001$ as compared to vehicle control group determined by Student's $t$ test.

RV-induced pDC-derived IFN production was dependent upon intact TLR7 signalling in vitro. Additionally, adoptive transfer of TLR7-expressing pDCs into allergic $\mathrm{Tlr}^{-/-}$hosts re-established type I and II IFN responses, limiting RV replication and eosinophilic airways inflammation. Notably TLR7 deficiency did not impair pDC recruitment into the lungs. This implicates activation of TLR7 signalling on pDCs and increasing host IFN release as important therapeutic
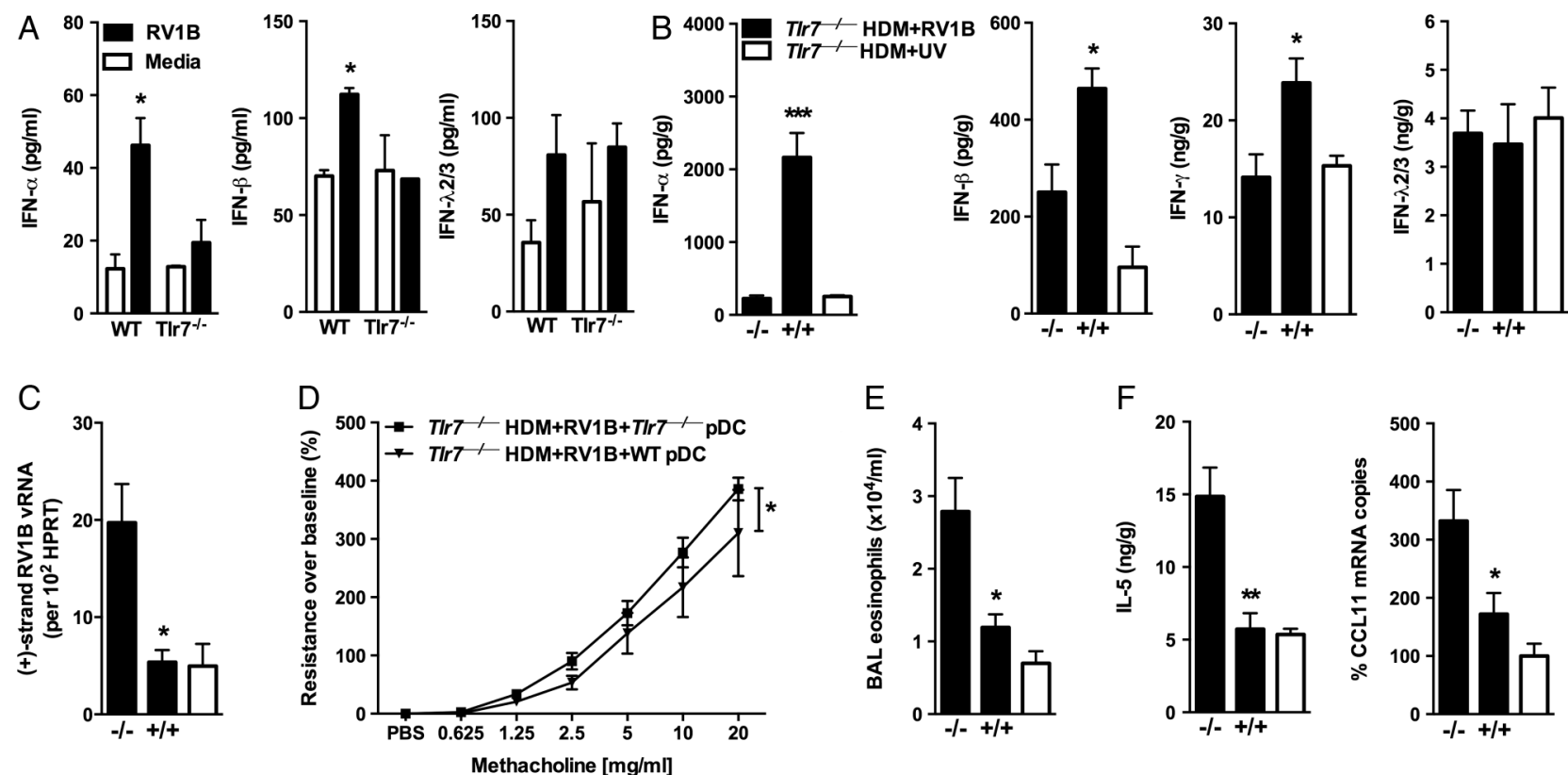

Figure 3 Adoptive transfer of Toll-like receptor (TLR)7-competent plasmacytoid dendritic cells ( $p D C s)$ to TLR7-deficient mice limits exacerbation of allergic airways disease (AAD). Purified Flt3-L-expanded pDCs from TLR7-deficient (-I-) and TLR7-competent (+/+) bone marrow were adoptively transferred to allergic $\mathrm{Tlr}^{-1-}$ recipients. Mice were inoculated with RV1B $2 \mathrm{~h}$ later and endpoints measured $24 \mathrm{~h}$ post infection. (A) Spleen-isolated pDCs were infected in vitro with RV1B and interferon (IFN) release in cell supernatants assessed by ELISA. Levels of IFNs (B), as well as interleukin (IL)-5 (F) in clarified lung homogenates as assessed by ELISA. Positive-strand RV1B RNA (C) and CCL11 expression (F) from lower airway tissue quantified by quantitative RT-PCR. (D) Total lung resistance presented as percentage change in response to methacholine ( $n=7-8$ mice per group). (E) Eosinophils present in bronchoalveolar lavage (BAL) fluid. Results are mean $\pm S E M\left(n=3-5\right.$ mice per group). ${ }^{*} p<0.05,{ }^{* *} p<0.01,{ }^{* * *} p<0.001$ determined by Student's t test except for (D) where analysis of variance compared the RI curves. All $p$ values as compared to exacerbated mice that received TLR7-deficient $\mathrm{pDCs}$. 

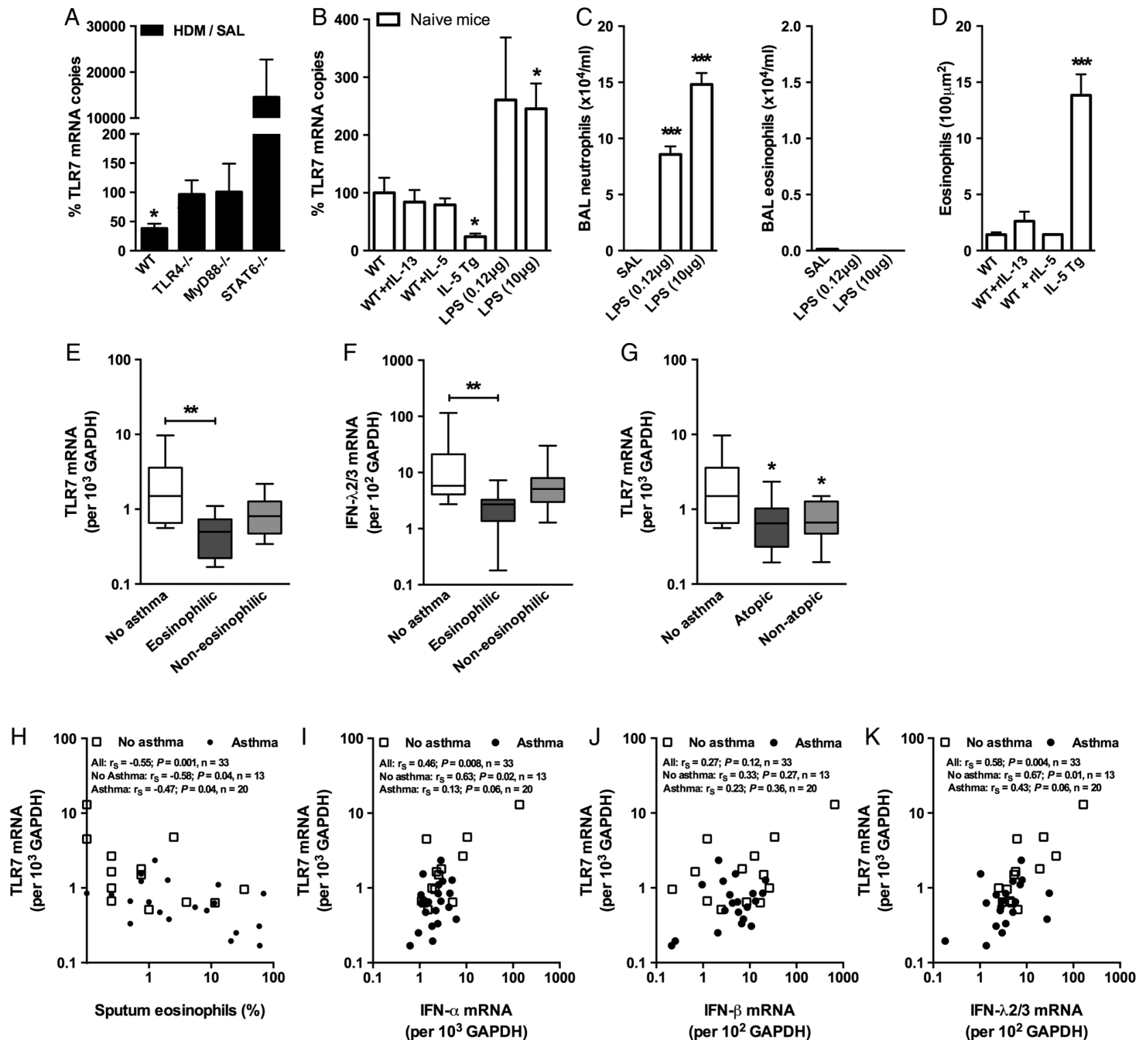

Figure 4 Toll-like receptor (TLR)7 expression is reduced during eosinophilic lung inflammation. (A) Wildtype (WT), TLR4 ${ }^{-1-}, M y D 88^{-1-}$ and Stat6 $6^{-1-}$ mice were sensitised and challenged with house dust mite (HDM) over 18 days and gene expression of TLR7 in lower airway tissue was quantified by quantitative RT-PCR. TLR7 airway expression in allergic airways as a percentage of sterile saline (SAL) expression for each strain, (B) as well as lung tissue eosinophils and (C) bronchoalveolar lavage (BAL) fluid neutrophils and eosinophils (D) from rlL-5, rlL-13, IL-5 Tg and lipopolysaccharide (LPS)-treated mice $24 \mathrm{~h}$ post treatment. Results are mean \pm SEM ( $n=4-6$ mice per group), gene expression in mice expressed as a \% compared to non-allergic SAL-treated wildtype mice. TLR7 (E) and interferon (IFN) $\lambda 2 / 3$ (F) expression in bronchial biopsies collected from non-asthmatic ( $n=13$ ) and asthmatic $(n=20)$ subjects stratified into eosinophilic ( $>3 \%$ eosinophils) or non-eosinophilic ( $<3 \%$ eosinophils) phenotypes based on BAL cell counts. (G) TLR7 expression in bronchial biopsies collected from non-asthmatic $(n=13)$ and asthmatic $(n=20)$ subjects stratified according to atopic status. Lines indicate the median, boxes extend from 25th to the 75th percentile, and error bars extend to 10th and 90th percentiles. (H) Correlation between TLR7 expression from bronchial biopsies and percentage of sputum eosinophils. (I-K) Correlations between TLR7 and IFN expression from patient biopsies. ${ }^{*} p<0.05,{ }^{* *} p<0.01,{ }^{* *} p<0.001$ as determined by Student's t test (A-D) or Kruskal-Wallis with Dunn's multiple comparisons test.

strategies, either through the use of TLR7 agonists or targeting pathways upstream of IFN production. Other IFN-producing cells may also be relevant. We have recently shown that anti-CCL7 treatment is sufficient to inhibit IRF-7-dependent IFN $\beta$ expression in RV infection, which was associated with reduced macrophage inflammation but not pDC influx. ${ }^{24}$ This highlights the complexity of the inflammatory and antiviral immune response on a cellular level, particularly in an allergic environment.

WT allergic mice with $\mathrm{T}_{\mathrm{H}}$ 2-driven $\mathrm{AAD}$ had significantly lower levels of TLR7 expression compared with non-allergic SAL-treated mice in the absence of RV infection, which is in line with the pattern seen clinically in non-infected eosinophilic asthmatics. TLR4 signalling is required for the development of a robust $\mathrm{T}_{\mathrm{H}} 2$-mediated allergic airways inflammation in response to HDM extract, which also contains low amounts of endotoxins. ${ }^{19}$ Allergic airways inflammation suppresses TLR7 expression, which is prevented by disruption of $\mathrm{T}_{\mathrm{H}} 2$-promoting signalling pathways governed by TLR4, MyD88 and STAT6. In contrast to allergic mice, TLR4 activation by LPS led to an upregulation of TLR7 expression in non-allergic mice. Thus the effects of TLR4 signalling on the regulation of TLR7 expression are determined by the presence or absence of $\mathrm{T}_{\mathrm{H}}$ 2-dominant allergic airways inflammation. Interestingly, LPS also upregulated TLR3 
Figure 5 Proposed role and regulation of Toll-like receptor (TLR)7 in rhinovirus infection and allergic airways disease. IL, interleukin; RV, rhinovirus.

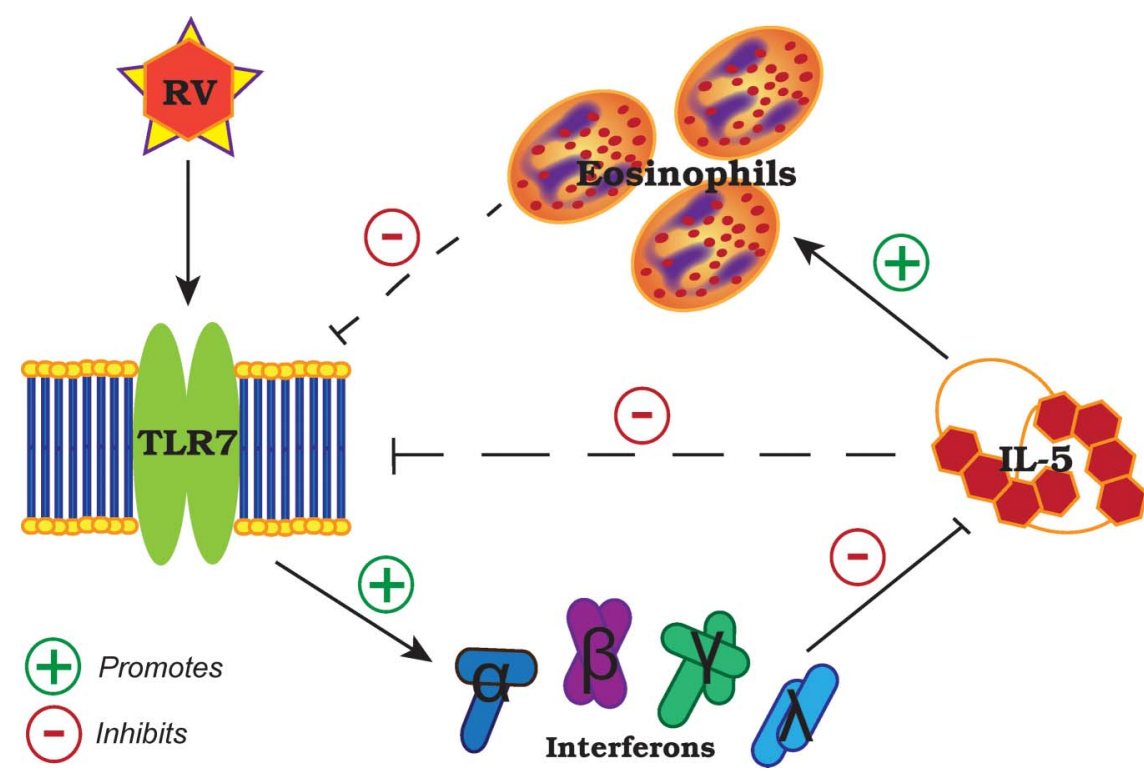

expression in human monocytes, which was critical for antiviral responses. $^{25}$ TLR3-/- mice infected with RV1B, however, displayed normal type I IFN responses, unchanged viral titres and reduced inflammatory responses. ${ }^{10}$ This is in marked contrast to our data generated in allergic TLR7-/ - mice and previous data in non-allergic TLR7-/- mice. ${ }^{20}$ Further studies are now required to elucidate the clinical effect of endotoxins on TLR7-mediated responses in asthma.

In the absence of $\mathrm{T}_{\mathrm{H}}$ 2-dominant allergic airways inflammation, no suppression of TLR7 expression was observed by a single exposure to IL-13 or IL-5. However, chronic exposure to IL-5 alone, which is associated with lung eosinophilia and the development of AHR, ${ }^{26}$ markedly impaired TLR7 lung expression in the absence of allergy. This finding was of clinical relevance because we observed reduced TLR7 expression in endobronchial biopsies from asthmatic patients with eosinophilic inflammatory profiles. Eosinophilic asthmatics also expressed lower levels of innate IFNs in addition to TLR7, a difference that was not observed for expression of TLR3, retinoic acid-inducible gene I or melanoma differentiation-associated gene $5 .^{27}$ These results are congruent with a recent study that mapped TLR7 expression in the airways of severe asthmatics. ${ }^{28}$

Our results suggest a reciprocal regulation between IL-5-induced eosinophilia and TLR7 expression that affects antiviral IFN responses to RV (summary illustrated in figure 5). In the absence of virus, it has been shown that TLR7 stimulation with synthetic agonists in vitro resulted in inhibition of IL-5 through IFN $\gamma$ and Notch signalling pathways in antigenpresenting cells ${ }^{29}$ and upregulation of IFN $\gamma$ production by memory CD4 $+\mathrm{T}$ cells ${ }^{30}$ and natural killer cells. ${ }^{31}$ Consistent with these findings, we show impaired IFN $\gamma$ release in the absence of TLR7 promotes $\mathrm{T}_{\mathrm{H}} 2$ immune responses, which can be rescued by type I and III IFN therapy or adoptive transfer of TLR7-sufficient pDCs. This is directly relevant to subjects with moderate-to-severe asthma with a predominantly eosinophilic airways inflammation as these individuals have suppressed-but not fully deficient-TLR7 expression in their lungs, which correlated with reduced IFN expression. Notably, a human monoclonal antibody to IL-5 (mepolizumab) reduced exacerbation frequency in moderate-to-severe asthmatics with a predominantly eosinophilic airways inflammation, ${ }^{32}$ a strategy that may be of specific benefit to that clinical population as not all asthmatics appear to exhibit impaired IFN responses to $\mathrm{RV}^{33}$ Our data suggest that TLR7 and its downstream signalling pathway limits $\mathrm{T}_{\mathrm{H}} 2$ responses in $\mathrm{RV}$-induced asthma exacerbations and may be a promising therapeutic target for the prevention and treatment of viral exacerbation in eosinophilic asthmatics.

\section{Author affiliations}

${ }^{1}$ Department of Experimental \& Translational Respiratory Medicine, Hunter Medical Research Institute, University of Newcastle, Newcastle, New South Wales, Australia ${ }^{2}$ Priority Research Centre for Asthma and Respiratory Diseases, Hunter Medical Research Institute, University of Newcastle, Newcastle, New South Wales, Australia ${ }^{3}$ Department of Respiratory and Sleep Medicine, John Hunter Hospital, Newcastle, New South Wales, Australia

${ }^{4}$ Norman Bethune Medical Science Centre, Jilin University, Jilin, Changchun, China

${ }^{5}$ The School of Biomedical Sciences, University of Queensland, Queensland,

Queensland, Australia

${ }^{6}$ Airway Disease Infection Section, National Heart and Lung Institute, Medical

Research Council \& Asthma UK Centre in Allergic Mechanisms of Asthma, Imperial College London, Norfolk Place, London, UK

${ }^{7}$ Department of Paediatric Respiratory and Sleep Medicine, Newcastle Children's Hospital, Newcastle, New South Wales, Australia

Acknowledgements We would like to thank S Akira, Osaka University, for providing TIr $4^{-I-}$, MyD $88^{-I-}$ and TLR $7^{-I-}$ mice and thank $L$ Dent for providing IL-5 $5^{T / T g}$ mice. We appreciate the technical assistance from A Pereira de Siqueira, C Cesar de Souza Alves, J Grehan, H Macdonald, M Morten, L Sokulsky and the staff from the animal care facility of the University of Newcastle.

Contributors $\mathrm{LH}$ and $\mathrm{AC}$ contributed equally, performed and designed mouse and cell culture experiments, analysed data, generated figures and edited the manuscript. $J G$ and JL performed experiments and analysed data. JZ assisted in supervision. PABW and KP performed and supervised studies on healthy subjects and subjects with asthma, collected and processed biopsies, and performed cell culture experiments. SP assisted in the design and conceptualisation of some mouse experiments. DK supervised and interpreted cell culture experiments. NWB and SLJ assisted in design of mouse experiments, provided RV1B for further propagation and CDNA standards. PSF assisted in design, supervision and interpretation of mouse studies. JM conceptualised, coordinated, designed and supervised mouse and human studies, interpreted and analysed data, and drafted and edited the manuscript. All authors contributed to data discussion and revised the manuscript.

Funding This study was supported by the National Health and Medical Research Council (NH \& MRC 1011153) (JM, PSF), the Hunter Medical Research Institute (JM, PABW, PSF), the Hunter Children's Research Foundation (JM, PSF) and an NH\&MRC Health Practitioner Research Fellowship to JM (455623). NWB was Asthma UK (CH1155). This work was supported in part by MRC Centre Grant G1000758 and ERC FP7 Advanced Grant 233015 (to SL).

Competing interests SLJ has received consulting fees from GlaxoSmithKline, Chiesi, Boehringer Ingelheim and Novartis. PABW and SLJ are authors on patents supported by a project grant from Asthma UK (06-050) and SLJ by a Chair from 
relating to use of IFNs in treatment of exacerbations of airway disease and holds share options in Synairgen, a company developing IFNs for treatment of exacerbations of airway disease.

\section{Patient consent Obtained.}

Ethics approval Hunter New England Area Health Service Ethics Committee and the University of Newcastle Committee Safety Committee (205/2008).

Provenance and peer review Not commissioned; externally peer reviewed.

Open Access This is an Open Access article distributed in accordance with the terms of the Creative Commons Attribution (CC BY 4.0) license, which permits others to distribute, remix, adapt and build upon this work, for commercial use, provided the original work is properly cited. See: http://creativecommons.org/ licenses/by/4.0/

\section{REFERENCES}

1 Holgate $S$, Roberts $G$, Arshad H, et al. The role of the airway epithelium and its interaction with environmental factors in asthma pathogenesis. Proc Am Thorac Soc 2009;6:655-9.

2 Weckmann M, Collison A, Simpson J, et al. Critical link between TRAIL and CCL20 for the activation of TH2 cells and the expression of allergic airway disease. Nat Med 2007;13:1308-15.

3 Wills-Karp M, Luyimbazi J, Xu X, et al. Interleukin-13: central mediator of allergic asthma. Science 1998;282:2258-61.

4 Mattes J, Yang M, Mahalingam S, et al. Intrinsic defect in T cell production of interleukin (IL)-13 in the absence of both IL-5 and eotaxin precludes the development of eosinophilia and airways hyperreactivity in experimental asthma. J Exp Med 2002;195:1433-44.

5 Wenzel SE. Asthma phenotypes: the evolution from clinical to molecular approaches. Nat Med 2012;18:716-25.

6 Johnston SL, Pattemore PK, Sanderson G, et al. Community study of role of viral infections in exacerbations of asthma in 9-11 year old children. BMJ 1995;310:1225-9.

7 Wark PA, Johnston SL, Bucchieri F, et al. Asthmatic bronchial epithelial cells have a deficient innate immune response to infection with rhinovirus. J Exp Med 2005;201:937-47.

8 Contoli M, Message SD, Laza-Stanca V, et al. Role of deficient type III interferon-lambda production in asthma exacerbations. Nat Med 2006:12:1023-6.

9 Sykes A, Edwards MR, Macintyre J, et al. Rhinovirus 16-induced IFN-alpha and IFN-beta are deficient in bronchoalveolar lavage cells in asthmatic patients. J Allergy Clin Immunol 2012;129:1506-14.

10 Wang Q, Miller DJ, Bowman ER, et al. MDA5 and TLR3 initiate pro-inflammatory signaling pathways leading to rhinovirus-induced airways inflammation and hyperresponsiveness. PLOS Pathog 2011;7:e1002070.

11 Ioannidis I, Ye F, McNally B, et al. TLR expression and induction of type I and type III interferons in primary airway epithelial cells. J Virol 2013;87:3261-70.

12 Ekman AK, Adner M, Cardell LO. Toll-like receptor 7 activation reduces the contractile response of airway smooth muscle. Eur J Pharmacol 2011;652:145-51.

13 Collison A, Hatchwell $\mathrm{L}$, Verrills $\mathrm{N}$, et al. The E3 ubiquitin ligase midline 1 promotes allergen and rhinovirus-induced asthma by inhibiting protein phosphatase $2 \mathrm{~A}$ activity. Nat Med 2013;19:232-7.

14 Koltsida 0, Hausding M, Stavropoulos A, et al. IL-28A (IFN-lambda2) modulates lung DC function to promote Th1 immune skewing and suppress allergic airway disease. EMBO Mol Med 2011;3:348-61.

15 Collison A, Li J, Pereira de Siqueira A, et al. Tumor necrosis factor-related apoptosis-inducing ligand regulates hallmark features of airways remodelling in allergic airways disease. Am J Respir Cell Mol Biol 2014;51:86-93.
16 Hatchwell L, Girkin J, Dun M, et al. Salmeterol attenuates chemotactic responses in rhinovirus-induced exacerbation of allergic airways disease by modulating protein phosphatase 2A. J Allergy Clin Immunol 2014;133:1720-7.

17 Collison A, Mattes J, Plank $\mathrm{M}$, et al. Inhibition of house dust mite-induced allergic airways disease by antagonism of microRNA-145 is comparable to glucocorticoid treatment. J Allergy Clin Immunol 2011;128:160-7.

18 de Souza Alves CC, Collison A, Hatchwell L, et al. Inhibiting AKT phosphorylation employing non-cytotoxic anthraquinones ameliorates TH2 mediated allergic airways disease and rhinovirus exacerbation. PLoS ONE 2013;8:e79565.

19 Mattes J, Collison A, Plank M, et al. Antagonism of microRNA-126 suppresses the effector function of TH2 cells and the development of allergic airways disease. Proc Natl Acad Sci USA 2009;106:18704-9.

20 Davidson S, Kaiko G, Loh Z, et al. Plasmacytoid dendritic cells promote host defense against acute pneumovirus infection via the TLR7-MyD88-dependent signaling pathway. J Immunol 2011;186:5938-48.

21 Gibson PG, Simpson JL, Saltos N. Heterogeneity of airway inflammation in persistent asthma: evidence of neutrophilic inflammation and increased sputum interleukin-8. Chest 2001;119:1329-36.

22 Roponen M, Yerkovich ST, Hollams E, et al. Toll-like receptor 7 function is reduced in adolescents with asthma. Eur Respir J 2010;35:64-71.

23 Kaiko GE, Loh Z, Spann K, et al. Toll-like receptor 7 gene deficiency and early-life Pneumovirus infection interact to predispose toward the development of asthma-like pathology in mice. J Allergy Clin Immunol 2013;131: 1331-9.

24 Girkin J, Hatchwell L, Foster PS, et al. CCL7 and IRF-7 mediate hallmark inflammatory and IFN responses following Rhinovirus 1B infection. J Immunol 2015; 194:4924-30.

25 Pan ZK, Fisher C, Li JD, et al. Bacterial LPS up-regulated TLR3 expression is critical for antiviral response in human monocytes: evidence for negative regulation by CYLD. Int Immunol 2011;23:357-64.

26 Lee JJ, McGarry MP, Farmer SC, et al. Interleukin-5 expression in the lung epithelium of transgenic mice leads to pulmonary changes pathognomonic of asthma. J Exp Med 1997;185:2143-56.

27 Parsons KS, Hsu AC, Wark PA. TLR3 and MDA5 signalling though not expression, is impaired in asthmatic epithelial cells in response to rhinovirus infection. Clin Exp Allergy 2014;44:91-101.

28 Shikhagaie MM, Andersson CK, Mori M, et al. Mapping of TLR5 and TLR7 in central and distal human airways and identification of reduced TLR expression in severe asthma. Clin Exp Allergy 2014;44:184-96.

29 Edwards S, Jones C, Leishman AJ, et al. TLR7 stimulation of APCs results in inhibition of IL-5 through type I IFN and Notch signaling pathways in human peripheral blood mononuclear cells. J Immunol 2013:190:2585-92.

30 Caron G, Duluc D, Fremaux I, et al. Direct stimulation of human T cells via TLR5 and TLR7/8: flagellin and R-848 up-regulate proliferation and IFN-gamma production by memory CD4+ T cells. J Immunol 2005;175:1551-7.

31 Hart OM, Athie-Morales V, O'Connor GM, et al. TLR7/8-mediated activation of human NK cells results in accessory cell-dependent IFN-gamma production. J Immunol 2005; 175:1636-42.

32 Pavord ID, Korn S, Howarth P, et al. Mepolizumab for severe eosinophilic asthma (DREAM): a multicentre, double-blind, placebo-controlled trial. Lancet 2012;380:651-9.

33 Sykes A, Macintyre J, Edwards MR, et al. Rhinovirus-induced interferon production is not deficient in well controlled asthma. Thorax 2014;69:240-6. 